\section{Relativity and Indeterminacy}

Wiтнобт wishing in any way to disagree with H. Bondi's view ${ }^{1}$ that "relativity demands a nondeterministic theory", I would suggest that his final summing-up is somewhat misleading. He says : "The flow of time has no significance in the logically fixed pattern of events demanded by deterministic theory, time being a mere co-ordinate. In a theory of indeterminacy, however, the passage of time transforms statistical expectations into real events".

In the case of deterministic theories, we must surely distinguish between reversible and irreversible determined processes. 'Time, in statistical but determined laws, as distinct from dynamical laws, surely has a very definite significance, for example, in its relation to entropy changes and especially to those processes which Prof. A. R. Ubbelohde ${ }^{2}$ has described as "disentropic". (These are defined as "happenings which turn against the trend towards increasing entropy".) Many statistical laws depend on determined processes. The motions of individual components may be difficult to observe, or too numerous or complex to calculate, yet their behaviour certainly falls outside the range of appreciable indeterminacy.

It is true, if not a truism, that where there is no cause-effect relation, real events become actual from mere statistical expectations during the course of time. But can this passage of time rightly be described as the transforming agent? This would involve a return to causality with time as the cause !

I would suggest that there is at least as much difference between time used as a co-ordinate in dynamical processes and time describing local variations in efficiency (both being determined) as there is between either of these and time during which (not by which) non-determined statistical probabilities become actualities.

\section{G. W. Scott BLaIR}

National Institute for Research in Dairying, University of Reading.

Bondi, H., Nature, 169, 660 (1952). "Ubbelohde: A. R., "Time and Thermodynamics", 97 (Oxf. Univ.

IN a recent communication in Nature ${ }^{1}$, Mr. $\mathrm{H}$. Bondi (in common with a large number of writers in the field) took as a fundamental postulate of relativity the principle $P$ that effects are delayed relative to causes by amounts corresponding to transmission speeds less than or equal to the velocity of light, $c$. This led him to the conclusion that indeterminacy is fundamental to relativity, and so we must abandon the complete determinacy contemplated in classical physics (and, it should be noted, in at least one approach to quantum theory ${ }^{2}$ ). The argument was, more or less, that there is no unique cause-effect link, and so no unique meaning for $P$, in a deterministic system.

The principle $P$ is commonly employed in setting up a system of space-time co-ordinates by means of light signals, and it appears that this is the use envisaged by Mr. Bondi. However, the important fact in such a co-ordinato formation is not that $c$ is the maximum speed between cause and effect, but rather that $c$ is a speed on which relatively moving observers will agree for all directions of motion.

One can set up one's own co-ordinate system in any number of ways, using light signals or not; all that is needed is a unique (to an arbitrary factor) and self-consistent method of correlating events which occur at separated spatial and temporal positions. Whether or not these events are related through a cause effect link is irrelevant and, some would say, meaningless.

If two correlated events occur at separations $\Delta r$ and $\Delta t$, then one can introduce an effective speed of correlation, $s=|\Delta r| \Delta t \mid$. This need not be the speed of a ponderable body nor of a light signal; it could just as well be, for example, a group speed or the speed of the juncture point of two straight edges in relative motion. It need not, then, be confined to values less than or equal to $c$. In this connexion, it may be pointed out that no escape, for example by considering discontinuous fields, need be made from the fact that group speeds can exceed $c$.

A difficulty arises only if we attempt to compare our measurements with those made, on the same events, in a relatively-moving co-ordinate system. In general, correlation is obtained by means of Lorentz, rather than Galilean, transformations, and in particular, the effective speeds are found equal (for all directions) in those cases when $s=c$ rather than when $s$ is infinite. It is these circumstances that give $c$ its unique position in the establishment of coordinate systems. Cause-effect links and limiting velocities are not involved, and conclusions based on them are therefore irrelevant to the fundamentals of relativity.

For those who may not agree with the foregoing, I shall add the following remarks. There are alternative, though less popular, methods of introducing relativity which postulate neither a cause-effect link nor limiting velocities. We are quite at liberty to adopt one of these other methods, and many would choose to do so. Mr. Bondi's apparent preference for $P$ does not commit the rest of us to indeterminacy, any more than Prof. P. A. M. Dirac's interpretation ${ }^{3}$ of his symbolism-whether it leads to quantization or not-commits us to an æther.

Department of Mathematics,

C. O. Hines

University of Cambridge.

1 Bondi, H., Nature, 169, 660 (1952).

- Bohm, D., Phys. Rer., 85, 166 (1952).

3 Dirac, P. A. M., Nature, 168, 906 (1951).

IT is not clear why the subject of statistics should throw any light on this problem, as Dr. G. W. Scott Blair suggests. 'There are two possible logical foundations for the use of statistics in physics: either, in the framework of a fully deterministic theory, wherein statistics may be used for the discussion of problems of such complexity that the detailed application of the supposedly known physical laws is impracticable; or within a non-deterministic theory, where the most basic physical laws may be concerned with the statement of probabilities.

In the former case, time is a co-ordinate, that, like space, is required for plotting the world lines of the particles. Whether this fixed pattern of world lines is simple so that we can construct it directly from the deterministic laws, or whether it is so complicated that we use statistical theorems as well as the deterministic laws in the calculation, does not alter the significance we attach to the various co-ordinates.

In the case of indeterminacy, the passage of time has a further significance, being concerned with the 